\title{
FEASIBILITY OF TUNABLE AMPLIFIER AND BANDPASS FILTER FOR MOBILE HANDSETS USING ACTIVE INDUCTOR CIRCUITS
}

\author{
J. RODRIGUEZ TELLEZ*, N.T. ALI ${ }^{\dagger}$ and B. MAJEED \\ Etisalat College of Engineering, P.O. Box 980, Sharjah, UAE
}

(Received 18 May 2002; In final form 3 July 2002)

\begin{abstract}
In this paper active inductor circuits are employed to assess their suitability for providing a tuning function in GaAs MMIC circuits. The specifications for a mobile handset amplifier and a bandpass filter operating from a $3 \mathrm{~V}$ supply rail are used as test vehicles. The design and simulation of the circuits employs a low-cost commercially available low pinch-off GaAs MESFET process. The suitability of active inductors for tuning in such applications considers issues such as frequency tuning range, noise, power consumption and stability.
\end{abstract}

Keywords: Active inductors; MMIC; GaAs MESFET

\section{INTRODUCTION}

In a previous paper [1], a number of active inductor circuit topologies were analysed and compared to assess their suitability for performing a tuning function in MMIC applications. The simulation work was based on a low-cost commercially available low pinch-off voltage GaAs MESFET process [2]. The low pinch-off voltage requirement enabled the circuits to function from a relatively low power supply rail $(<3 \mathrm{~V})$. This was believed to be an important consideration in the context of mobile communication handset applications. Issues such as inductance range, inductance bandwidth, series resistance and power consumption figured highly in this initial work for the same reasons. The simulation work, whilst employing reasonably accurate models for the active devices in the circuits did not consider the effect which the parasitic components introduced by the layout and passive components would have on the figures of merit mentioned above. The work also did not consider the practicality of using such inductors in a typical application.

In this paper, we describe the problems encountered in the utilisation of the active inductors when applied to an amplifier and filter design. For continuity these two circuit applications employ the same GaAs MESFET low pinch-off voltage process.

\footnotetext{
* Corresponding author.

† E-mail: ntali@ece.ac.ae
} 


\section{AMPLIFIER/FILTER SPECIFICATIONS AND TECHNOLOGICAL DATA}

The specifications for the amplifier and filter are shown in Table I. The centre frequency whilst specified at $1.8 \mathrm{GHz}$ was treated as a variable since the idea of the work is to assess how much tuning can be achieved with the active inductors. Generally speaking the tuning range does not need to be large for the idea to be useful. A tuning capability large enough to compensate for processing variations or to compensate for the poor threshold control of FET based circuits would be highly attractive. The specifications quoted do not necessarily correspond to a particular mobile system and are only intended to serve as a test vehicle.

The design and analysis process was performed with the HP-MDS design system [3] using the GEC-Marconi F20 process library. This process library contains accurate microwave models to represent the active, passive and interconnect components of a circuit. A detailed description of these models can be found in Refs. [2] and [3]. It is worth noting that because of convergency difficulties with the analysis of active inductors a nonlinear simulation approach could not be adopted. The analysis of these circuits under different bias conditions was performed with the linear simulator in the HP-MDS system. Although this approach is more time-consuming it yielded results which can be trusted to a higher extent. This analysis approach also enabled the active devices of the circuits to be simulated using their measured $s$-parameters. Clearly the simulation of the circuits at different bias points required the parameters of the linear MESFET model or the $s$-parameters of the device to be evaluated separately for each bias point. This approach is clearly only applicable because the operation of the amplifier and filter with active inductors was assumed to be quasi-static.

\subsection{Conventional Amplifier}

The single-stage amplifier circuit topology selected for this work is shown in Figure 1. This circuit configuration and the design procedure are well established. The amplifier specifications could of course be achieved with other circuit topologies but the one selected serves as a good vehicle for illustrating some of the limitations of the use of active inductors.

Of the devices available in the F20 process library, the six-finger $150 \mu \mathrm{m}$ gate-width/finger ( $900 \mu \mathrm{m}$ total gate-width) low pinch-off $\left(V_{p}=-1 \mathrm{~V}\right)$ MESFET was selected as being the most suitable for the amplifier. Biased for maximum efficiency at $20 \%$ of the maximum current and a drain-source voltage $\left(V_{D S}\right)$ of $3 \mathrm{~V}$, the dc power consumption was $129 \mathrm{~mW}$. With these bias conditions the input and output matching components were optimised to satisfy the specifications at $1.8 \mathrm{GHz}$. With the bias conditions to the device and the capacitor values kept constant, the tuning range was then explored by adjusting the inductance values.

TABLE I Amplifier and Filter Specifications.

\begin{tabular}{lcc}
\hline & Amplifier & Filter \\
\hline Centre frequency (MHz) & 1842 & 1842 \\
Frequency range (MHz) & $1710-1880$ & $1805-1880$ \\
Input/output reflection coefficient (dB) & $\leq 15$ & - \\
Gain (dB) & $\geq 15$ & - \\
DC power rail (V) & 3 & 3 \\
Noise figure (dB) & $<4$ & - \\
Insertion loss (dB) & - & 2.5 \\
Return loss in bandwidth (dB) & - & 10 \\
Ripple in bandwidth (dB) & - & $25\left(f_{c} \pm 140 \mathrm{MHz}\right)$ \\
Attenuation relative to insertion loss $(\mathrm{dB})$ & - & \\
\hline
\end{tabular}




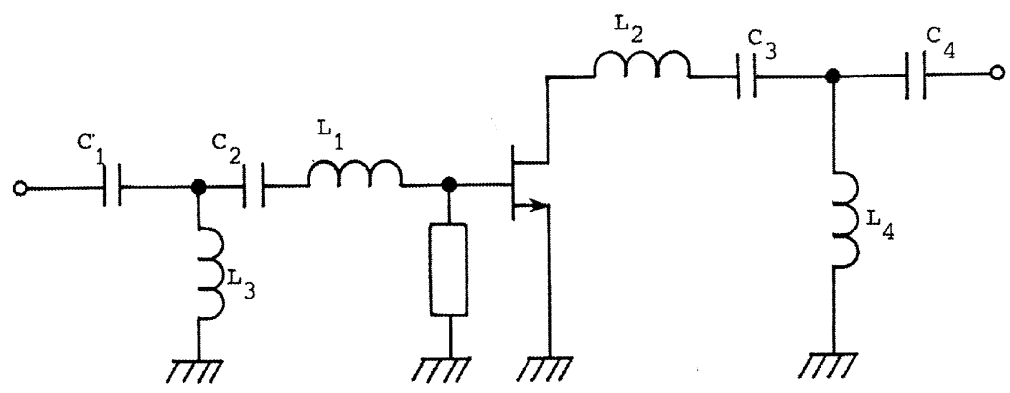

FIGURE 1 Amplifier circuit topology (device is $900 \mu \mathrm{m}$ gate-width MESFET).

This showed that the amplifier could be tuned over a wide frequency range and still meet the same specifications at different frequencies. Table II shows the matching element values at $900 \mathrm{MHz}, 1.8 \mathrm{GHz}$ and $2.5 \mathrm{GHz}$ with the device biased as stated above. For the three frequencies specified, the stability criteria was met by employing a $260 \Omega$ shunt resistance as shown in Figure 1. Although the results shown in Table II do not take into account interconnect effects, it nevertheless gives a good indication of the inductance range required from the active inductors. Such a broad frequency tuning range especially the lower frequency area, whilst useful, may however be unnecessary in the case of the mobile handset market where the trend seems to be for higher frequency of operation.

\subsection{Conventional Filter}

The circuit topology selected to fulfil the bandpass filter specifications was a third-order Tchebyshev filter as shown in Figure 2. As with the amplifier, this circuit and the design procedures have been extensively documented. Unlike the amplifier however, it was soon determined that the frequency range over which the filter could be tuned solely by altering the inductances was not as large as for the amplifier. In fact the same frequency span could only be achieved by altering the capacitor values as well as the inductances as is shown in Table III. These results do not take into account interconnect effects and, unlike the amplifier design considered previously, the passive components were represented as ideal components. The use of more realistic models for the passive elements, especially the inductors, yielded very poor performance due to the losses or poor $Q$ of the components. Although the need for variable capacitors could be overcome by utilising active capacitors,

TABLE II Conventional Amplifier Matching Element Values (Capacitor Values Constant and Optimised at $1842.5 \mathrm{MHz}$ ).

\begin{tabular}{lccc}
\hline & At $902.5 \mathrm{MHz}$ & At $1842.5 \mathrm{MHz}$ & At $2.5 \mathrm{GHz}$ \\
& $L_{1}=14.47 \mathrm{nH}$ & $L_{1}=4.26 \mathrm{nH}$ & $L_{1}=2.16 \mathrm{nH}$ \\
& $L_{2}=15.28 \mathrm{nH}$ & $L_{2}=5.31 \mathrm{nH}$ & $L_{2}=3.31 \mathrm{nH}$ \\
& $L_{3}=9.47 \mathrm{nH}$ & $L_{3}=2.12 \mathrm{nH}$ & $L_{3}=1.32 \mathrm{nH}$ \\
& $L_{4}=17.29 \mathrm{nH}$ & $L_{4}=4.45 \mathrm{nH}$ & $L_{4}=2.67 \mathrm{nH}$ \\
& -16 & -19 & -13 \\
& -27 & -20 & -18 \\
$S_{11}(\mathrm{~dB})$ & 16 & 17 & 17 \\
$S_{12}(\mathrm{~dB})$ & -16 & -16 & -12 \\
$S_{21}(\mathrm{~dB})$ & 2.9 & 4.2 & 4.3 \\
$S_{22}(\mathrm{~dB})$ & & & \\
Noise figure (dB) & & & \\
\hline
\end{tabular}

$C_{1}=6.35 \mathrm{pF} ; C_{2}=6.41 \mathrm{pF} ; C_{3}=3.24 \mathrm{pF} ; C_{4}=3.67 \mathrm{pF}$. 


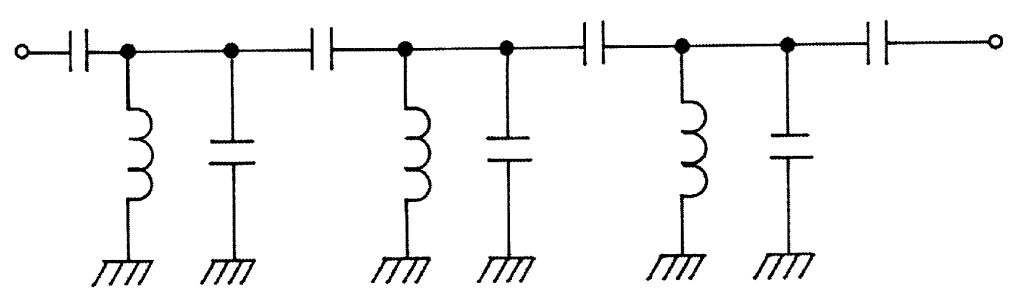

FIGURE 2 Third-order Tchebyshev conventional filter.

this aspect is beyond the scope of this paper. The active filter design will therefore concentrate on the higher of the two frequency bands shown in Table III since this is more relevant to existing and possible future mobile systems.

\section{THE ACTIVE TUNING ELEMENTS}

Extensive trials using the active inductors described in Ref. [1] showed that for the amplifier the best performance was achieved with the cascode-based Khoury active inductor [4] shown in Figure 3. This inductor was previously shown to offer reasonably wide bandwidth and broad inductance range. Unfortunately at moderately high frequencies the series resistance of the circuit changes from a positive to a negative value which leads to stability problems. This particular problem was overcome by providing additional resistance (can also be capacitance or both) feedback to stabilise the series resistance of the circuit at some small but positive value as shown in Figure 3. This resistance value is only effective over a modest frequency range. In some situations therefore this resistance may need to be implemented with a FET to achieve a variable bias controlled resistance. This would enable moderately high $Q$ values to be achieved at different frequency points whilst maintaining stable operation. This modification to the inductor circuit is clearly also beneficial from a noise point of view since this is related to the series resistance associated with the circuit.

The inductance value obtained from the circuit shown in Figure 3 is principally determined by the gate-bias applied to transistor $T_{c}$. The gate-bias applied to the other devices also influence the inductance value but to a lesser degree. These transistors are therefore normally

TABLE III Conventional Filter Component Values for 2 Frequency Ranges.

\begin{tabular}{|c|c|c|c|c|}
\hline & $\begin{array}{c}L_{r 1}, L_{r 2} \\
L_{r 3}=10 n H\end{array}$ & $\begin{array}{c}L_{r 1}, L_{r 2}, \\
L_{r 3}=2.5 n H\end{array}$ & $\begin{array}{c}L_{r 1}, L_{r 2} \\
L_{r 3}=10 n H\end{array}$ & $\begin{array}{c}L_{r 1}, L_{r 2}, \\
L_{r 3}=5.45 n H\end{array}$ \\
\hline $3 \mathrm{~dB}$ bandwidth (MHz) & $890-916$ & $1822-1852$ & $1806-1881$ & $2456-2537$ \\
\hline Insertion loss $(\mathrm{dB})$ & 0 & 0.43 & 0 & 0 \\
\hline Return loss in BW (dB) & 10 & 9.8 & 10 & 15.6 \\
\hline Ripple in BW (dB) & 0.5 & 0 & 0.5 & 0.123 \\
\hline \multirow{2}{*}{$\begin{array}{l}\text { Attenuation relative } \\
\text { to IL }(\mathrm{dB})\end{array}$} & 21 & 85 & 115 & 37 \\
\hline & \multicolumn{2}{|c|}{$\begin{array}{c}C_{c 1}, C_{c 4}=0.448 \mathrm{pF} \\
C_{c 2}, C_{c 3}=0.067 \mathrm{pF} \\
C_{r 1}, C_{r 3}=2.6 \mathrm{pF} \\
C_{r 2}=2.974 \mathrm{pF} \\
\text { Optimised for operation } \\
\quad \text { at } 902.5 \mathrm{MHz}\end{array}$} & \multicolumn{2}{|c|}{$\begin{array}{c}C_{c 1}, C_{c 4}=0.182 \mathrm{pF} \\
C_{c 2}, C_{c 3}=0.023 \mathrm{pF} \\
C_{r 1}, C_{r 3}=0.543 \mathrm{pF} \\
C_{r 2}=0.7 \mathrm{pF} \\
\text { Optimised for operation } \\
\quad \text { at } 1842.5 \mathrm{MHz}\end{array}$} \\
\hline
\end{tabular}




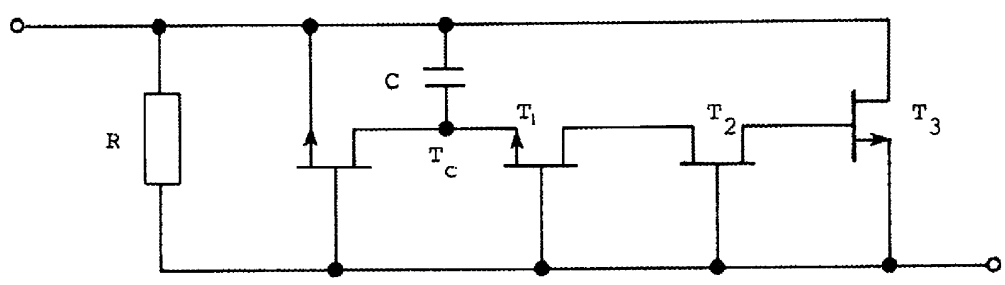

FIGURE 3 Active inductor with resistive and capacitive feedback.

biased at a $V_{G S}$ of zero volts. All the devices operate in the saturation region at a $V_{D S}$ of $2 \mathrm{~V}$. Table IV summarises the predicted inductance and series resistance of the circuit as a function of gate-bias to transistor $T_{c}$ at three frequency points of interest. This table also shows the inductance and series resistance of the circuit when the gate-width of the transistors of the circuit is varied. As can be seen, the inductance value realised increases as $V_{G S}$ approaches the pinch-off point. Unfortunately, the series resistance also increases and the bandwidth of the circuit decreases. Since the behaviour of the device parameters as $V_{G S}$ is varied is the same as when the device gate-width is varied, the same effect is seen when the gate-width is altered. The higher inductance values are therefore realised with smaller gate-width devices. As will be seen later the dependency of the inductance value on device size causes problems in applications where a high inductance value is required to pass a high current to a seriesconnected device. It should be noted that the results shown in Table IV do not incorporate the additional resistor required to prevent the series resistance of the inductor from becoming negative. The required values for each application will be presented later in the paper.

The results shown in Table IV and those presented in Ref. [1] also do not incorporate the parasitic components introduced by the passive components and layout. In this respect active inductors have been found to be unduly sensitive to such effects. Figure 4 for example compares the response of the inductor circuit of Figure 3 with and without parasitic effects. As can be seen, the parasitics increase the real and imaginary component of the circuit. Their main effect however is to considerably reduce the resonant frequency. Simulation results indicate that the most serious degradation to the inductors bandwidth is caused by shunt capacitive effects to ground. The main contribution in this respect arises from the size of the blocking capacitors needed to isolate the transistors from a dc point of view rather than from the tracks themselves whose effects can be minimised by careful design. Simulation results using the same circuit layout but with HEMTs $\left(f_{T}=40 \mathrm{GHz}\right)$ rather than MESFETs $\left(f_{T}=20 \mathrm{GHz}\right)$ showed little change in the inductor's resonant point,

TABLE IV Inductance and Series Resistance of Inductor as a Function of Gate-Bias $\left(\mathrm{T}_{\mathrm{c}}\right)$ and Device Gate-Width Size.

\begin{tabular}{lccccc}
\hline Bias & $\begin{array}{c}\text { Device } \\
\text { size }(\mu \mathrm{m})\end{array}$ & $\begin{array}{c}\text { Power } \\
(\mathrm{mW})\end{array}$ & $902.5 \mathrm{MHz}$ & $1842.5 \mathrm{MHz}$ & $2500 \mathrm{MHz}$ \\
\hline$V_{G S}=0 \mathrm{~V}$ & 25 & 7.7 & $9.32 \mathrm{nH},-1.9 \Omega$ & $10.5 \mathrm{nH},-12.82 \Omega$ & $12.28 \mathrm{nH},-25.57 \Omega$ \\
$V_{G S}=-0.4 \mathrm{~V}$ & & & $23.21 \mathrm{nH}, 2.28 \Omega$ & $30.35 \mathrm{nH}, 6.64 \Omega$ & $42.68 \mathrm{nH}, 94.64 \Omega$ \\
$V_{G S}=0 \mathrm{~V}$ & 50 & 15.4 & $4.74 \mathrm{nH},-0.78 \Omega$ & $5.29 \mathrm{nH},-5.19 \Omega$ & $6.04 \mathrm{nH},-10.05 \Omega$ \\
$V_{G S}=-0.4 \mathrm{~V}$ & & & $12.06 \mathrm{nH}, 2.01 \Omega$ & $15.23 \mathrm{nH}, 7.77 \Omega$ & $20.29 \mathrm{nH}, 50.18 \Omega$ \\
$V_{G S}=0 \mathrm{~V}$ & 100 & 30.8 & $2.25 \mathrm{nH},-0.24 \Omega$ & $2.43 \mathrm{nH},-1.76 \Omega$ & $2.66 \mathrm{nH},-3.37 \Omega$ \\
$V_{G S}=-0.4 \mathrm{~V}$ & & & $5.49 \mathrm{nH}, 1.2 \Omega$ & $6.38 \mathrm{nH}, 3.46 \Omega$ & $7.62 \mathrm{nH}, 12.78 \Omega$ \\
$V_{G S}=0 \mathrm{~V}$ & 300 & 92.4 & $0.77 \mathrm{nH},-0.13 \Omega$ & $0.85 \mathrm{nH},-0.8 \Omega$ & $0.95 \mathrm{nH},-1.55 \Omega$ \\
$V_{G S}=-0.4 \mathrm{~V}$ & & & $1.93 \mathrm{nH}, 0.3 \Omega$ & $2.34 \mathrm{nH}, 1 \Omega$ & $2.97 \mathrm{nH}, 5.56 \Omega$ \\
\hline
\end{tabular}



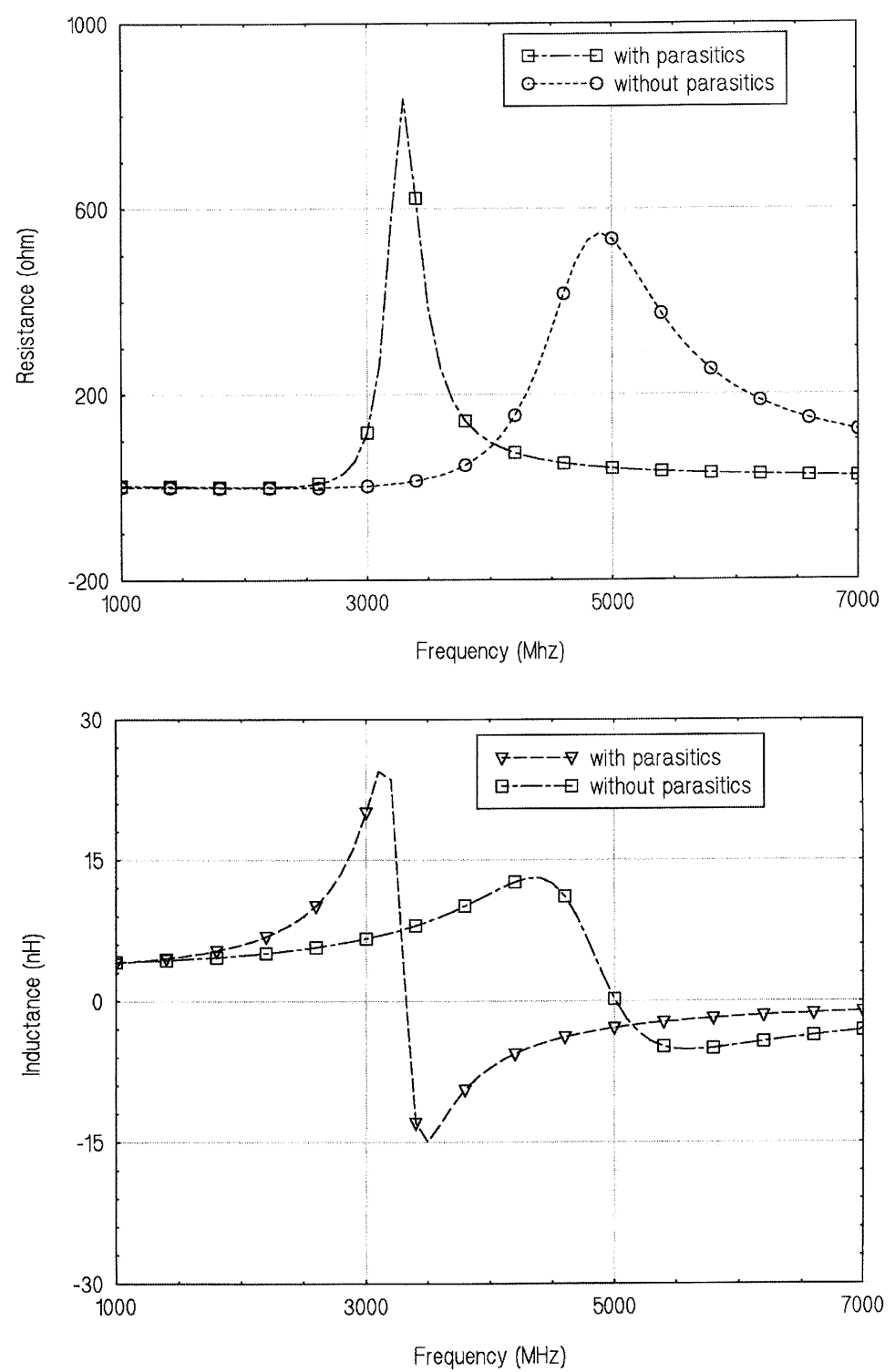

FIGURE 4 Series resistance and inductance of Khoury inductor.

indicating the circuit to be very much interconnect-limited. For the results shown in Figure 4, the dc blocking capacitors employed were $4.92 \mathrm{pF}$ which corresponds to capacitors of $100 \mu \mathrm{m}$ by $100 \mu \mathrm{m}$ in size. This size component has a total shunt capacitance to ground of $0.02 \mathrm{pF}$. For the inductor circuit 4 such capacitors are required for isolation. For the layout employed to yield the results shown in Figure 4, the total shunt capacitance to ground was $0.54 \mathrm{pF}$ which is considerably larger than the capacitance arising from the active devices themselves. In the amplifier and filter layout, the dc blocking capacitors were made as small as possible to reduce these shunt effects whilst at the same time providing adequate $\mathrm{dc}$ isolation. Although the resonant frequency of the inductor could be improved by altering the bias conditions to the devices to reduce the degradation introduced by the layout, the resulting improvements are small. 


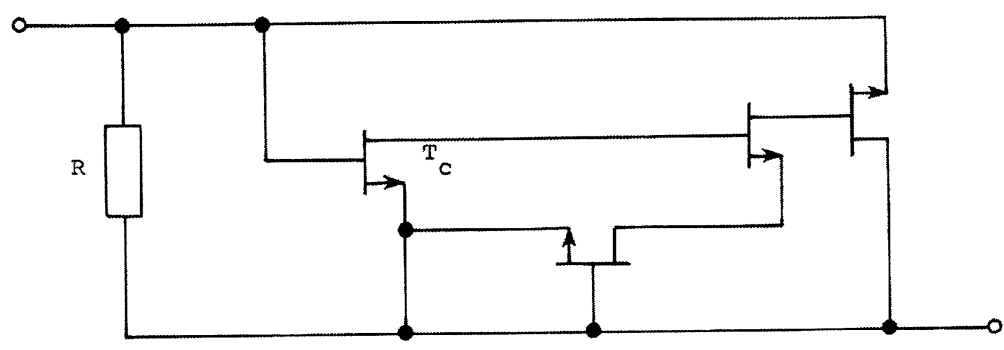

FIGURE 5 Zhang active inductor with resistive feedback.

Similar trials using the filter as a test vehicle revealed that the best performance was obtained with the cascode version of the Zhang lossless inductor [5] shown in Figure 5. The series resistance of this inductor also exhibits the same negative resistance dependency on frequency as the Khoury inductor described earlier. To circumvent the stability problems that this causes, additional resistive feedback was introduced, as shown in Figure 5. Since the behaviour of this inductor with respect to bias, frequency, gate-width size and susceptibility to parasitic effects is similar to the Khoury inductor, data concerning these aspects are not presented here. Further information on this and other inductor topologies can be found in Ref. [1].

\section{THE TUNABLE AMPLIFIER}

From the results presented earlier, the first obstacle which became evident is that the inductance values required for $L_{1}$ and $L_{2}$ to tune the amplifier over the frequency range specified requires device sizes which are too small and not supported in the GaAs process chosen. Even if this were not true, in the case of $L_{2}$ such a small size device may not be able to support the necessary power from the larger $900 \mu \mathrm{m}$ amplifying device. The replacement of $L_{1}$ for an active inductor would also be inadvisable due to the increase in the noise figure. This would rise from $4.1 \mathrm{~dB}$ for the conventional amplifier to $8 \mathrm{~dB}$ when $L_{1}$ is replaced by an active inductor implemented with $100 \mu \mathrm{m}$ devices. Because of these problems $L_{1}$ and $L_{2}$ were retained as conventional passive inductors with values corresponding to operation at $1.8 \mathrm{GHz}$. Clearly this reduces the tuning range considerably since only the two shunt inductors $L_{3}$ and $L_{4}$ are retained for this purpose. This however is unavoidable if the eventual performance is to be reasonably close to the specifications quoted. The layout for the tunable amplifier therefore employed two active shunt-connected inductors and these were implemented with $100 \mu \mathrm{m}$ devices.

The layout for the amplifier is shown in Figure 6. This chip is approximately $2874 \mu \mathrm{m}$ by $798 \mu \mathrm{m}$ in size. The six-finger $150 \mu \mathrm{m}$ gate-width/finger amplifying device and the twofinger $50 \mu \mathrm{m}$ gate-width/finger device employed for the active inductors are clearly visible. $120 \mu \mathrm{m}$ size contact pads are used for both the rf signal as well as for providing de bias to the device. Notice that in this prototype sufficient pads have been used to enable the bias conditions to all the devices to be individually adjusted. In its final form, this aspect could be simplified. For example, only the gate-bias to the controlling transistor $T_{c}$ of each active inductor needs to be accessed for tuning purposes.

The $s$-parameters of the laid-out amplifier in two frequency bands are shown in Figure 7. The two plots show the maximum frequency over which the amplifier could be tuned and still provide what could be considered respectable performance. For these results only the gatebias to the control transistor $\mathrm{T}_{\mathrm{c}}$ of each inductor was varied. For the input and output 


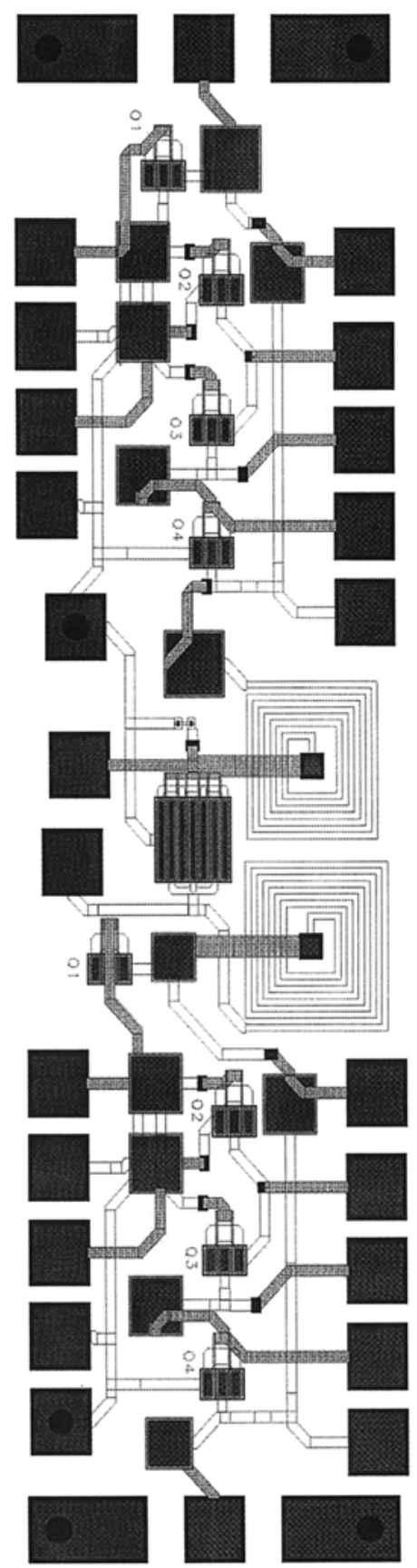

FIGURE 6 Layout of tunable amplifier.

shunt active inductors, resistances of $500 \Omega$ and $590 \Omega$ respectively were used to compensate for the negative series resistances of the inductor. It is interesting to note that the reason for the lower than expected $S_{21}$ is the resistance associated with the passive inductors and tracks, rather than the effects of the active inductors. The results show that with only two active inductors a tuning range of approximately $\pm 100 \mathrm{MHz}$ can be achieved. Although this is 

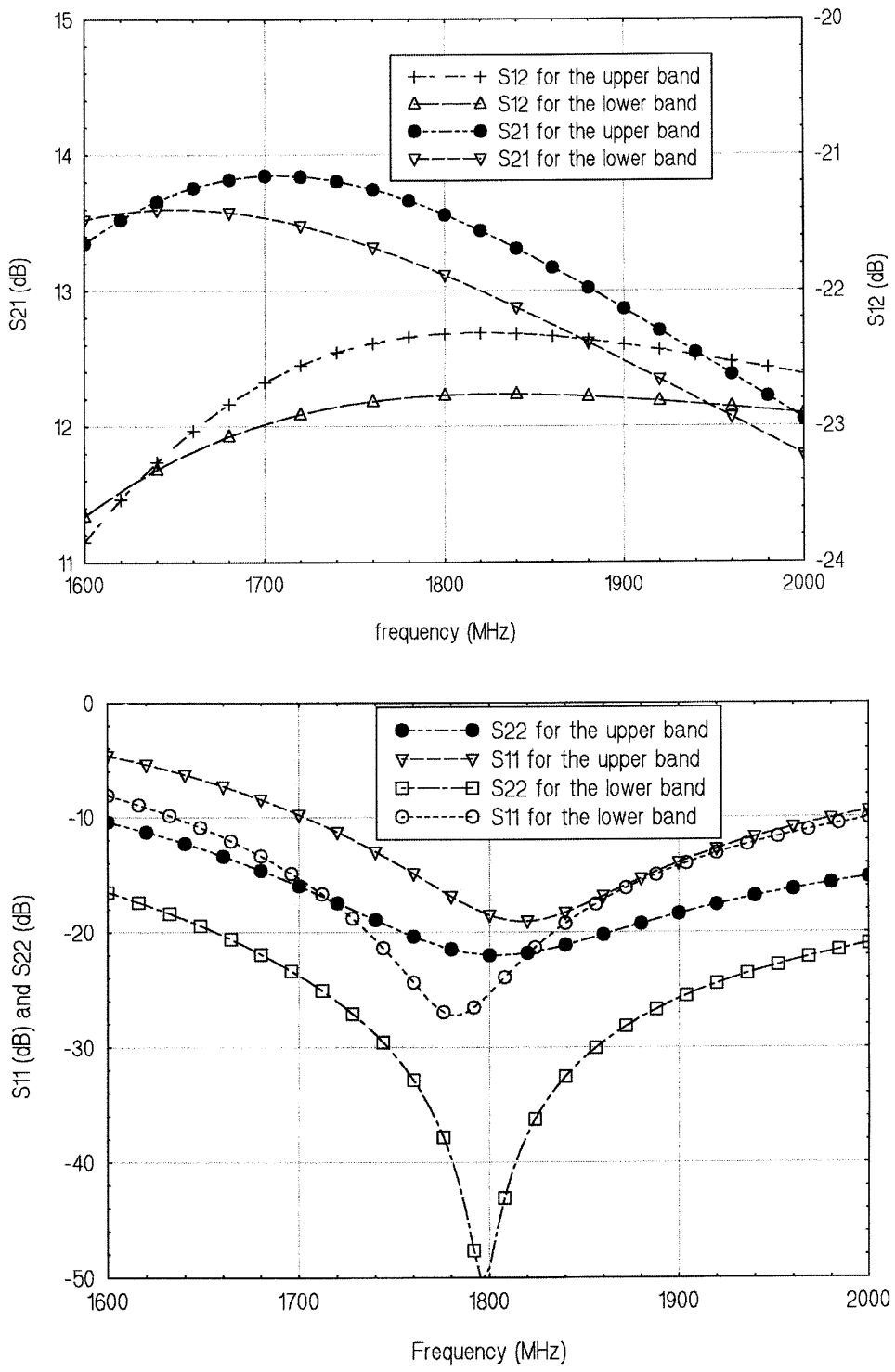

FIGURE 7 Scattering parameters for laid-out tunable amplifier.

too small to enable a reconfigurable handset to be developed for existing and third generation systems, it does enable the effects of processing variations to be compensated for.

For the tunable amplifier the total dc power consumption is $195 \mathrm{~mW}$ compared with $129 \mathrm{~mW}$ for the conventional amplifier. At $1842 \mathrm{MHz}$ the noise figure for the laid-out tunable amplifier was simulated as $4.3 \mathrm{~dB}$ and this compares favourably with the $4.1 \mathrm{~dB}$ noise figure of the laid-out conventional amplifier. At $1842 \mathrm{MHz}$ the $1 \mathrm{~dB}$ compression point for the conventional and tunable laid-out amplifiers were $6.2 \mathrm{dBm}$ and $1 \mathrm{dBm}$ respectively. In the case of the tunable amplifier the confidence level of this figure is not high since the active inductors cannot be simulated from a nonlinear point of view. The stability figure for the tunable laid-out amplifier was simulated as 1.89 at $1842.5 \mathrm{MHz}$. At $1742.5 \mathrm{MHz}$ this stability figure reduces to 1.46 . 


\section{THE TUNABLE FILTER}

The tuning of the third-order Tchebyshev filter was implemented with the cascode Zhang lossless active inductor. This inductor provides an inductance range from $18 \mathrm{nH}$ $\left(V_{G S}\right.$ of $\mathrm{T}_{\mathrm{c}}$ at $\left.-0.8 \mathrm{~V}\right)$ to $2 \mathrm{nH}\left(V_{G S}\right.$ of $\mathrm{T}_{\mathrm{c}}$ at $\left.+0.6 \mathrm{~V}\right)$ when implemented with $100 \mu \mathrm{m}$ gate-width devices operating at a $V_{D S}$ of $2 \mathrm{~V}$. This inductance range is sufficient to study the tuning range of the filter. The negative series resistance of the inductor was compensated for by introducing additional resistance feedback as described previously. Unlike the amplifier however the filter was found to be more sensitive to resistance effects and the feedback resistance of each inductor needed to be adjusted for operation at different frequencies. For operation at a centre frequency of $902.5 \mathrm{MHz}$ for example the feedback resistance required was $780 \Omega$ and this provided inductors with $Q s$ of 8200. For operation at a centre frequency of $1842.5 \mathrm{MHz}$ the feedback resistance required was $24.5 \Omega$ which yielded inductors with $Q s$ of 20,000. In practice, achieving such performance figures from the inductors would be very difficult. Although the simulation results presented later for the filter at these frequencies employed a passive resistor in its final form, this would be implemented with a FET to facilitate the tuning control.

The layout of the filter is shown in Figure 8. This chip is approximately $3260 \mu \mathrm{m}$ by $1580 \mu \mathrm{m}$ in size. The two-finger $50 \mu \mathrm{m}$ gate-width/finger devices of the inductors are clearly visible. As with the amplifier, sufficient contact pads were included to enable the bias conditions to the devices to be adjusted. In its final form the number of such pads can be reduced since it is only the gate-bias to the controlling transistor $T_{c}$ and the feedback resistor (when implemented with a FET) which needs to be accessed. Notice also that the layout of each inductor has been duplicated. This layout was arrived at after extensive optimisation. As with the amplifier considered previously, the resonant frequency of the inductor is very much determined by shunt parasitic effects introduced by the interconnect and more importantly the dc isolation capacitors. As with the amplifier layout, the rf chokes (or FETs) to isolate the rf from the dc supply lines may be implemented off-chip. This aspect is still under consideration.

The $s$-parameters of the laid-out filter in two frequency bands is shown in Figure 9. The two plots show the maximum frequency range over which the filter could be tuned and still provide what could be considered respectable performance. For these results only the gate-bias to the controlling transistors $T_{c}$ of each inductor and their feedback resistance was varied. The noise figure of the laid-out filter was, not surprisingly, found to be highly dependent on the operating conditions of the active inductors. For operation at a centre frequency of $902.5 \mathrm{MHz}$ the noise figure of the filter in the passband was $10 \mathrm{~dB}$. For operation at a centre frequency of $1842.5 \mathrm{MHz}$ the noise figure of the filter in the passband was $1.33 \mathrm{~dB}$. These noise figures almost entirely arise from the active inductors. The power consumption of the complete tunable filter is also clearly dependent on the operating conditions of the active inductors. At $902.5 \mathrm{MHz}$ and $1842.5 \mathrm{MHz}$ the de power consumption was $150 \mathrm{~mW}$ and $77 \mathrm{~mW}$ respectively. The $1 \mathrm{~dB}$ compression point of the filter could not be evaluated due to convergency difficulties, but we believe that this may be similar to the amplifier since the transistor sizes used for the active inductors are the same. As expected, the stability figure for the filter was very close to unity in the passband due to the very high $Q s$ required from the inductors. When implemented the series resistance of the inductors may need to be compromised to avoid stability problems. This however would reduce the $Q$ and worsen the noise performance. Results from the fabricated amplifier and filter will be published at a later date. 


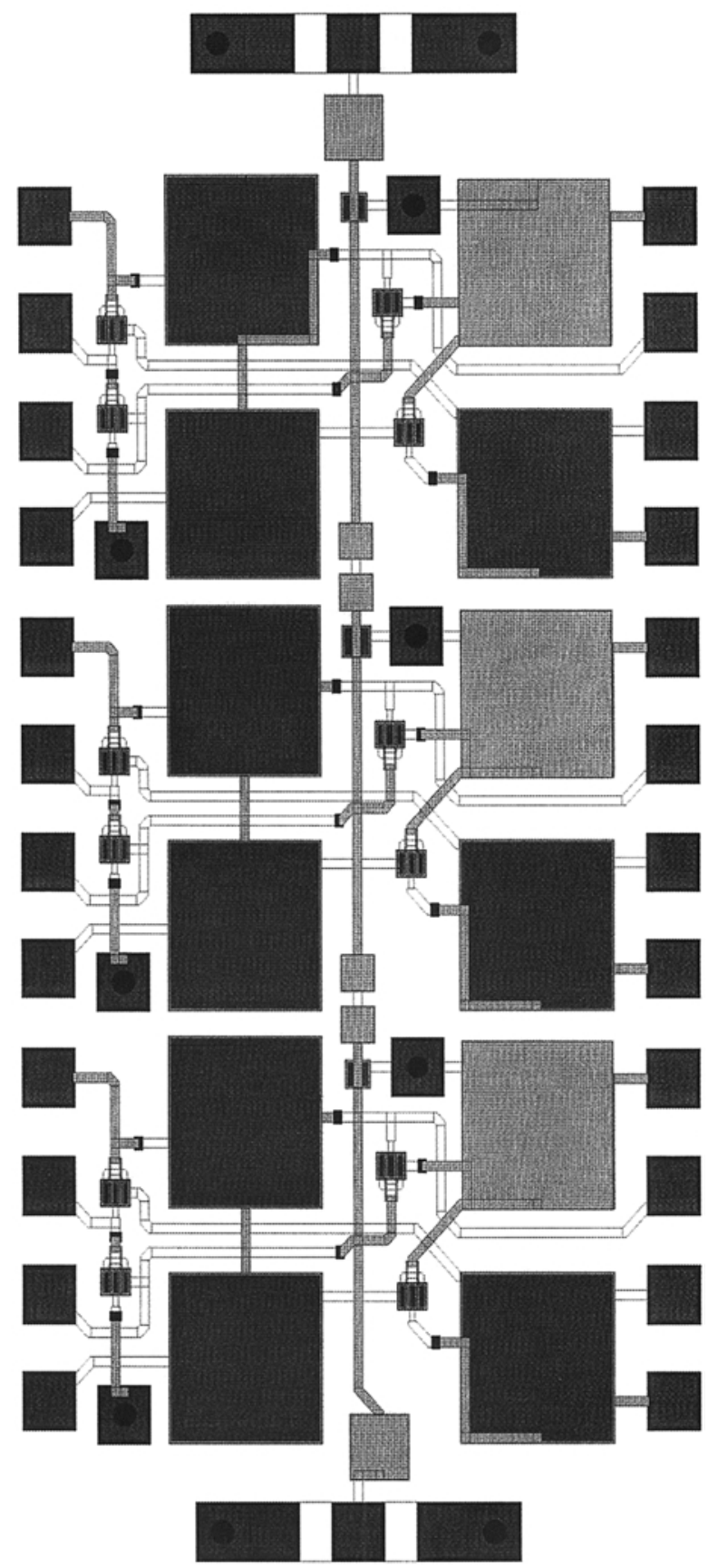

FIGURE 8 Laid-out tunable filter.

Although the figures quoted are in some respects unsatisfactory, the behaviour of the active filter described here is considerably better than can be achieved with an all-passive design. This is mainly due to the poor $Q$ factor of the available passive inductors. An $18 \mathrm{nH}$ inductor from the F20 process library for example has a series resistance of $18 \Omega$ at $2000 \mathrm{MHz}$ giving a $Q$ of 12.5 . 

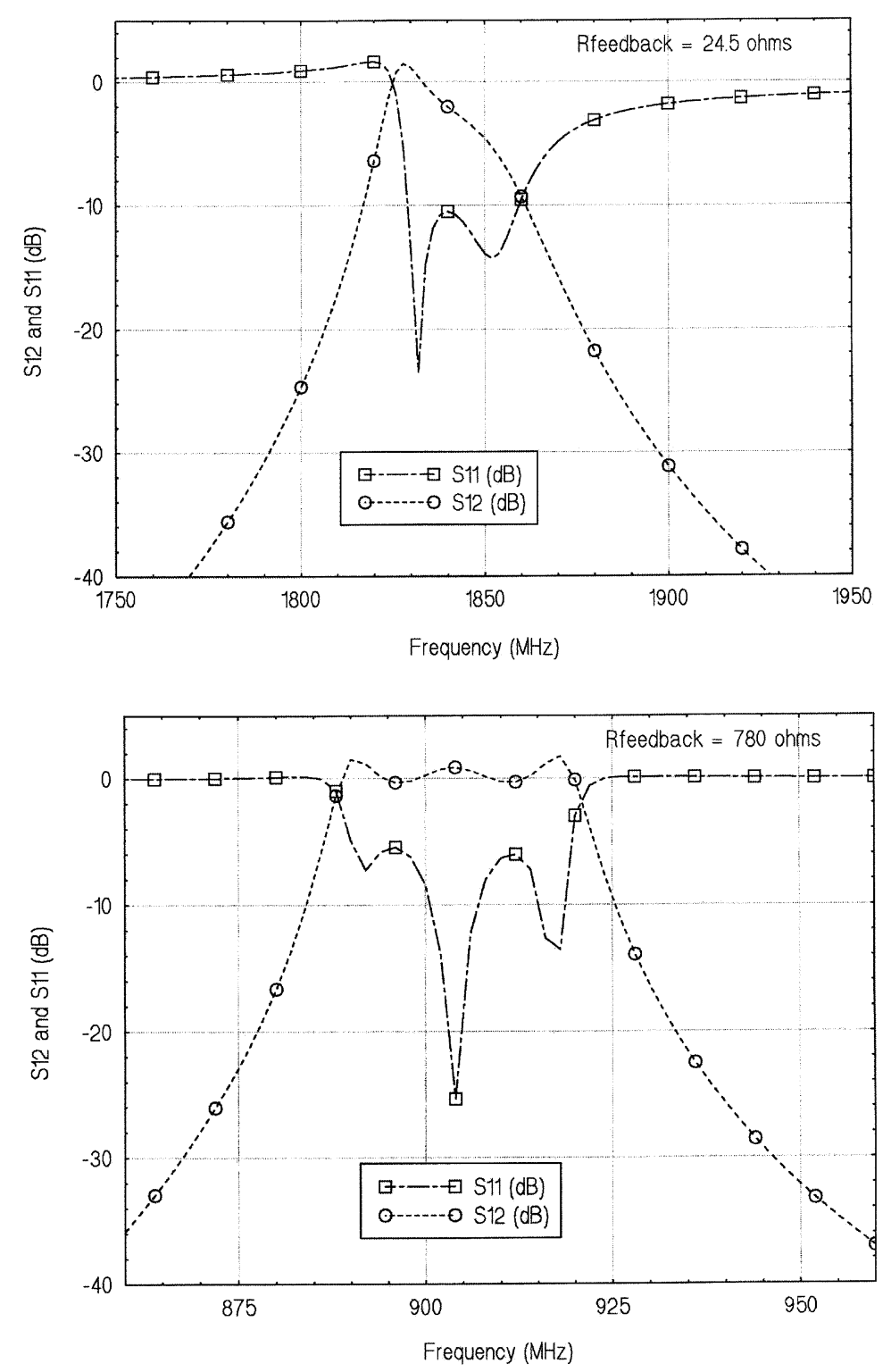

FIGURE $9 s$-parameters of laid-out tunable filter.

\section{SUMMARY}

Specifications for a mobile handset filter and amplifier have been used as a test vehicle to investigate the feasibility of employing active inductors as a means of introducing a tuning function in such circuits. The main points to have emerged from this initial work are

(i) The relationship between device size and inductance range can lead to a conflict in series connected applications between the current which such an inductor can carry and the inductance value required. This has been an issue in the amplifier design considered here. 
(ii) The stability problems caused by the negative series resistance of the active inductor circuits used have been overcome by introducing additional resistive feedback. By careful selection this can be used to yield a low but positive series resistance for the inductor circuit. In the two applications considered here, this has also been found to be an important consideration from a noise and $Q$ point of view.

(iii) Simulation results indicate that active inductor circuits are very much limited by interconnect and shunt effects to ground, rather than from the performance of the FET devices themselves. A comparison of a MESFET and a HEMT based inductor circuit shows a similar resonant point. The HEMT based design however enables the same inductance value to be realised with a smaller gate-width device.

(iv) Due to topological and inductor constraints, a tuning range of $\pm 100 \mathrm{MHz}$ centred around $1.8 \mathrm{GHz}$ has been realised for the amplifier considered here. Although this is not as large as was originally anticipated it is beneficial in many demanding applications where processing or poor threshold of the FET devices poses design difficulties. The noise $(4.2 \mathrm{~dB})$ and power consumption $(195 \mathrm{~mW})$ figures obtained however are poorer than a conventional amplifier.

(v) In the case of the bandpass filter a tuning range from $1700-2600 \mathrm{MHz}$ centred around $1.8 \mathrm{GHz}$ with an insertion loss of $0.019 \mathrm{~dB}$ and a $3 \mathrm{~dB}$ bandwidth of $67 \mathrm{MHz}$ has been realised. Again a useful tuning range which can overcome design and processing uncertainties. In power critical applications, however, the power consumption and noise figure need further assessment. Like the amplifier the filter operates from a $3 \mathrm{~V}$ power supply rail using a commercially available low-cost MESFET process.

The simulation results presented here, whilst employing industry-standard microwave models to represent as many features of the circuits as possible should be viewed with caution. The validity of the simulation results will hopefully be validated and reported soon, once the circuits are fabricated. The attractiveness of the idea and its application in other areas has hopefully been demonstrated.

\section{Acknowledgements}

The authors would like to thank T Pensato from the University of Bologna (Italy) for his valuable contribution to the filter design and FA Tosantos from the University of Cantabria (Spain) for his contribution to the amplifier design.

\section{References}

[1] Rodriguez Tellez, J., Diaz Asua, E. and Arinez, D. (1997). Assessment of active microwave inductors. IEE Proc. Circuits \& Devices Systems, 144(3), 161-166.

[2] GaAs IC Foundry Design Manual (Process F20/F14), GEC Marconi Materials Technology, Caswell, Towcester, NN12 8EQ, UK.

[3] Hewlett-Packard Series IV Microwave Design System. Hewlett-Packard Ltd, Stockport SK3 0RB, UK.

[4] El-Khoury, S. (1996). New approach to the design of active floating inductors in MMIC technology, IEEE Trans Microwave Theory \& Techniques, 44(4), 505-512.

[5] Zhang, G. F. and Gautir, J. L. (1993). Broadband lossless monolithic microwave active floating inductor. IEEE Microwave Guid. Wave Lett., 3(4), 98-100. 

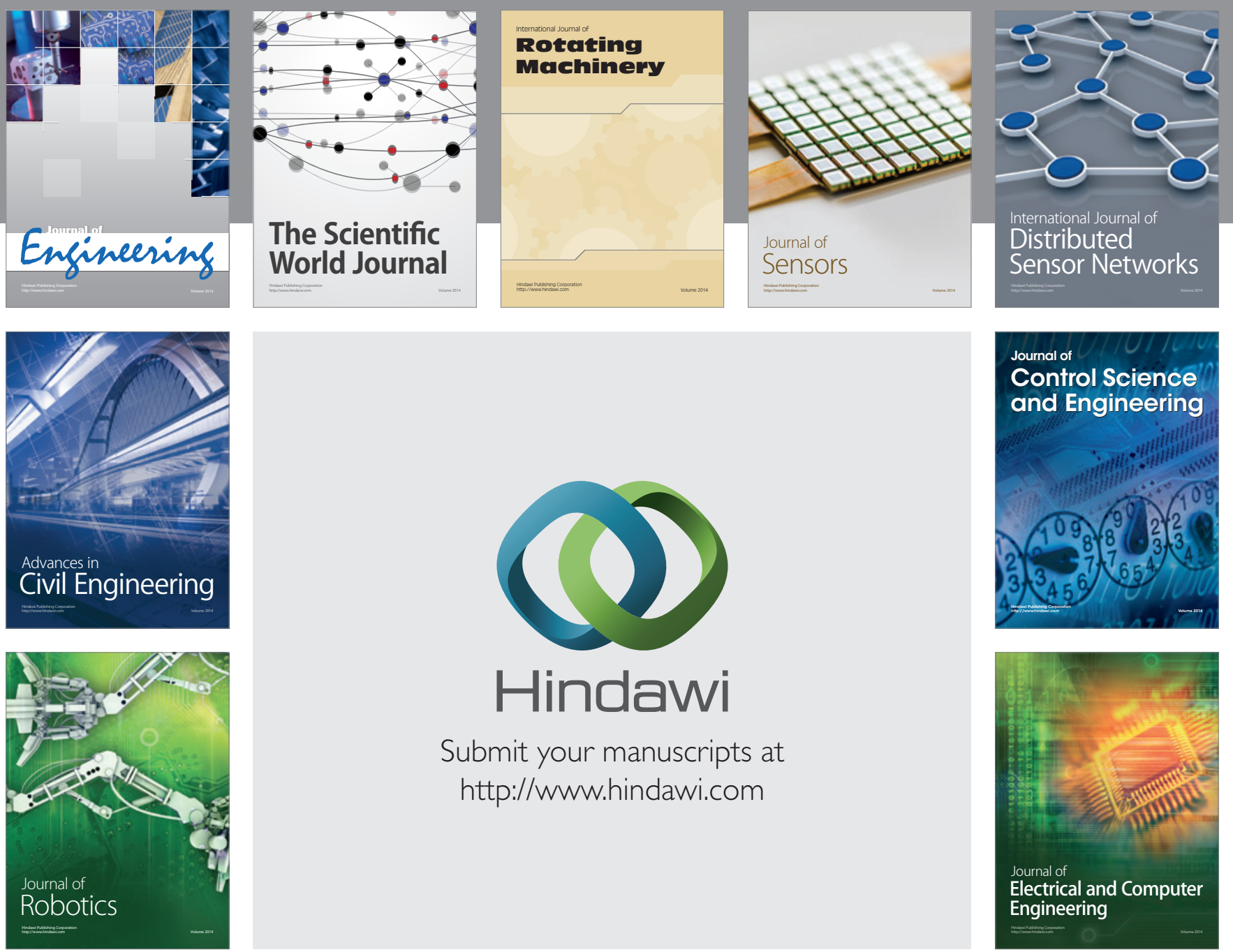

Submit your manuscripts at

http://www.hindawi.com
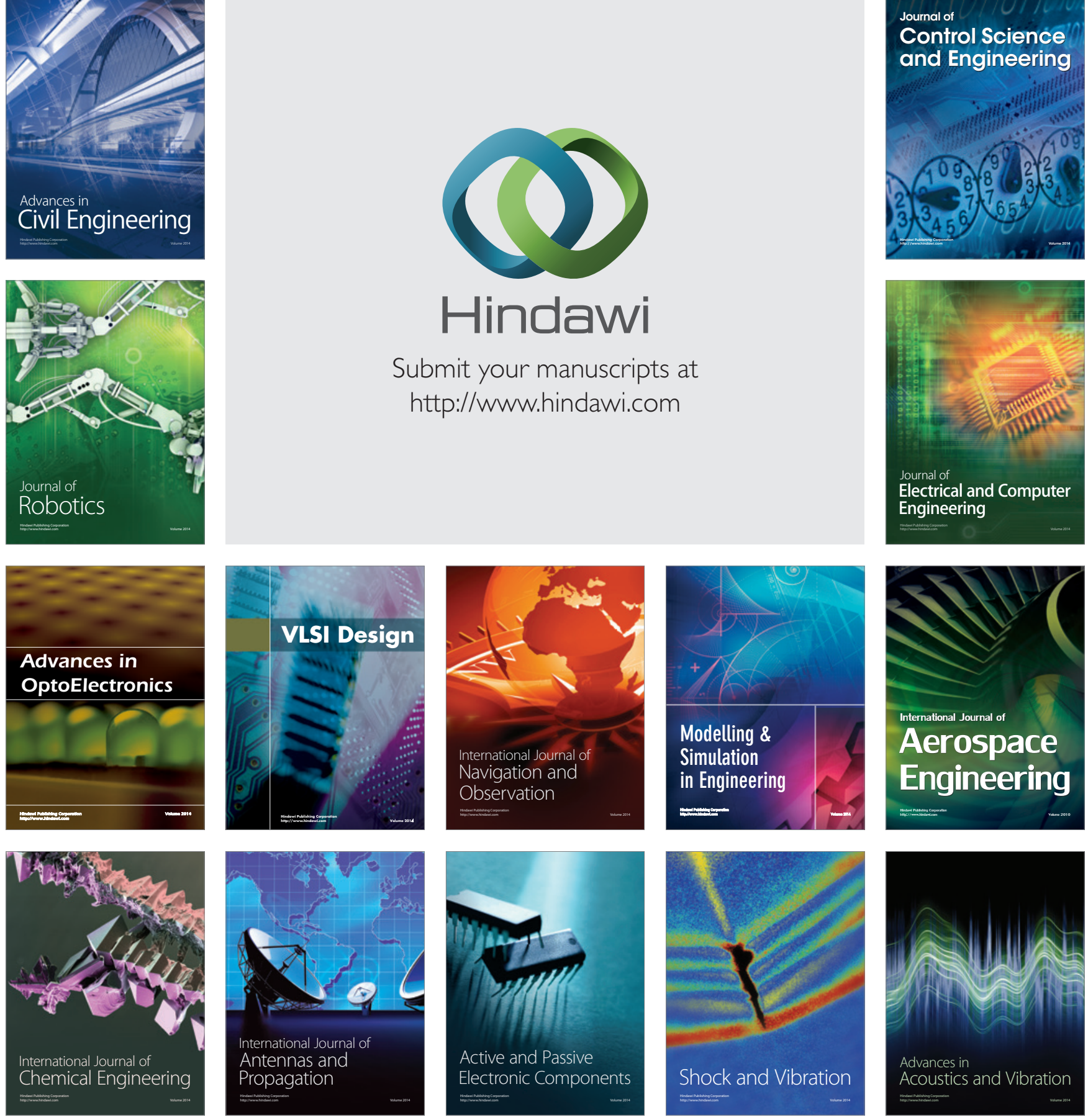